\title{
A survival analysis comparing women with ovarian low-grade serous carcinoma to those with high-grade histology
}

This article was published in the following Dove Press journal:

OncoTargets and Therapy

16 October 2014

Number of times this article has been viewed

\author{
Ming Chen' \\ Ying Jin' \\ Yalan $\mathrm{Bi}^{2}$ \\ Jie Yin' \\ Yongxue Wang' \\ Lingya Pan' \\ 'Department of Obstetrics and \\ Gynecology, Peking Union Medical \\ College Hospital, Chinese Academy \\ of Medical Sciences and Peking Union \\ Medical College, Beijing, People's \\ Republic of China; ${ }^{2}$ Department of \\ Pathology, Peking Union Medical \\ College Hospital, Chinese Academy \\ of Medical Sciences and Peking Union \\ Medical College, Beijing, People's \\ Republic of China
}

\begin{abstract}
Ovarian low-grade serous carcinoma (LGSC) and high-grade serous carcinoma have distinct molecular profiles, clinical behaviors, and treatment responses. The survival advantage for patients with low-grade carcinoma compared with patients with high-grade histology remains controversial. We retrospectively reviewed the medical charts of 381 patients with ovarian serous carcinoma at Peking Union Medical College Hospital from 2007 to 2010. Patients were classified into two groups according to MD Anderson two-tier system: 35 (9.2\%) cases with LGSC and 346 with high-grade serous carcinoma. Patients with low-grade serous ovarian cancer had a significantly younger age at diagnosis (46 versus 56 years, $P=0.046$ ), and their median progression-free survival (PFS) and overall survival values were 35.0 and 54.0 months, respectively. A multivariate analysis showed that, for serous ovarian cancer, the histological grade was a significant prognostic factor for PFS but not for overall survival $(P=0.022$ and $P=0.0566$, respectively). When stratified by the existence of a residual disease, patients with low-grade disease who underwent cytoreductive surgery without macroscopic residual disease $(>1 \mathrm{~cm})$ had a significantly improved median PFS time (36.0 months) compared with that of patients with high-grade carcinoma who received optimal cytoreductive surgery (16.0 months, $P=0.017)$. Conversely, patients with low-grade and high-grade carcinoma who were left with macroscopic residue $(>1 \mathrm{~cm})$ experienced a similarly shorter median PFS (10.0 and 13.0 months, respectively, $P=0.871$ ). The International Federation of Gynecology and Obstetrics stage and residual disease were significant prognostic factors of low-grade carcinoma, while positive ascites was associated with a worse PFS value. Our data showed that LGSC is a different entity from high-grade carcinoma and that LGSC was associated with improved PFS after optimal cytoreductive surgery but not suboptimal operation.
\end{abstract}

Keywords: ovarian carcinoma, low-grade serous carcinoma, high-grade serous carcinoma, prognosis

\section{Introduction}

Ovarian cancer is ten times less frequent than breast cancer, but it is associated with significantly more deaths. Approximately $75 \%$ of patients with epithelial ovarian cancer present with advanced (stage III-IV) tumors and experience recurrence after the standard treatment; most of these patients ultimately die of the disease. ${ }^{1}$ Currently, epithelial ovarian cancer is no longer considered to be a homogeneous disease worldwide; rather, it is recognized as a group of diseases that can be classified based on distinctive morphologic and molecular genetic features. ${ }^{2}$ Serous adenocarcinoma (SAC) is the most common histotype of epithelial ovarian cancer. Moreover, its low- and high-grade subtypes can be classified into two distinct types of ovarian cancer according to Kurman's dualistic model. ${ }^{3}$ 
A growing body of research demonstrates that significant differences exist in the molecular and clinical characteristics of low-grade and high-grade serous carcinomas. Low-grade serous carcinoma (LGSC) represents less than $10 \%$ of all cases of serous ovarian carcinomas. LGSC tends to be confined to the ovary and to behave in an indolent manner, most likely arises in a stepwise way from borderline tumors, and has a few copy number abnormalities but exhibits activating mutations of Ras, Raf, and PTEN genes. High-grade serous carcinoma (HGSC) is characterized by more aggressive behavior. Most HGSCs have mutations in the $p 53$ gene, and $40 \%$ also show mutations or epigenetic alterations in $B R C A$ genes. ${ }^{4,5}$ Several studies have demonstrated that LGSC is associated with better prognoses than other forms of serous ovarian cancer. ${ }^{6,7}$ Despite these data, several reports suggest that women with the low-grade disease exhibit chemotherapy resistance and remain at high risk for recurrence and cancerrelated death. ${ }^{8} 9$ Furthermore, few studies concerning the characteristics of or prognoses associated with LGSC have been conducted in Asian populations. Thus the survival outcome of LGSC is still controversial. Moreover, few comparison studies stratify serous cancer based on the two-tier MD Anderson Cancer Center (MDACC) system, which was proposed by Malpica in 2004 to counter the traditional threetier Shimizu-Silverberg system. ${ }^{10-12}$ This grading system subdivides tumors into low and high grade, based primarily on the assessment of nuclear atypia, with mitotic rate used as a secondary feature. It has been used in the MDACC for more than 15 years, was successfully validated in follow-up studies, and was shown to be easy to learn and highly reproducible among general and gynecologic pathologists.

This cohort study retrospectively reviewed the medical files of patients with LGSC and those of high-grade controls at Peking Union Medical College Hospital, a tertiary teaching hospital in the People's Republic of China, from 2007 to 2010. Additionally, the two-tier MDACC system was used to discriminate the two subtypes. We sought to determine the prognostic value of clinicopathological variables among women with low-grade serous ovarian cancer and to compare the outcomes between those with low-grade and high-grade disease.

\section{Patients and methods}

We retrospectively reviewed the medical charts of patients with epithelial ovarian cancer who were treated and received complete surgical staging or cytoreductive surgery at the Division of Gynecological Oncology within the Department of Obstetrics and Gynecology at Peking Union Medical
College Hospital, Peking Union Medical College, People's Republic of China. This study included 381 patients with ovarian serous carcinoma who were admitted between January 2007 and December 2010. Moreover, this study obtained the University Institutional Review Board approval from Peking Union Medical College and all participants provided written informed consent. All patients with stage I and II tumors received completed staging surgery; women with advanced (stage III and IV) cancer underwent optimal cytoreduction, except those with unresectable tumors who received suboptimal cytoreductive surgery (CRS). All women received adjuvant chemotherapy containing platinum and taxane after primary surgery. Carboplatin was calculated as the area under the curve $=6$, and cisplatin was administered at $75 \mathrm{mg} / \mathrm{m}^{2}$. Paclitaxel was administered at $175 \mathrm{mg} / \mathrm{m}^{2}$.

The follow-up time ranged from 11 to 82 months. The follow-up assessments of the patients consisted of a clinical examination, a blood test to determine tumor markers (carbohydrate antigen (CA)125, carcino-embryonic antigen (CEA), and CA199), and imaging studies including ultrasonographic scan and computed tomography (if necessary). After completing primary treatment (surgery and chemotherapy, if necessary), patients were examined every 3 months for the first 2 years, every 6 months for the next 3 years, and yearly thereafter. Recurrence was defined as a clinical relapse based on the National Comprehensive Cancer Network ovarian cancer guidelines. Progression-free survival (PFS) was calculated as the number of months from the completion of primary treatment to the date of clinical recurrence. Overall survival (OS) was defined as the number of months from the completion of primary treatment to the date of death.

Two senior pathologists at our hospital reviewed all pathology slides. A third reviewer evaluated any discordance. The pathologic diagnosis of low-grade and high-grade serous adenocarcinoma was made based on the MDACC two-tier system. This system is based primarily on the assessment of nuclear atypia, with mitotic rate used as a secondary feature. ${ }^{11}$ Tumors with mild-to-moderate cytologic atypia were designated as low-grade, whereas tumors with marked cytologic atypia were designated as high-grade (Figure 1). Grade 2 $\mathrm{SAC}$ in the conventional grading system corresponds to a high grade in the MDACC two-tier system. ${ }^{12}$

For statistical analysis, patients were classified into low-grade and high-grade groups using the MDACC twotier system. We also analyzed median age and menopausal status at diagnosis of ovarian cancer, the Eastern Cooperative Oncology Group performance status, the distribution of the International Federation of Gynecology and Obstetrics 

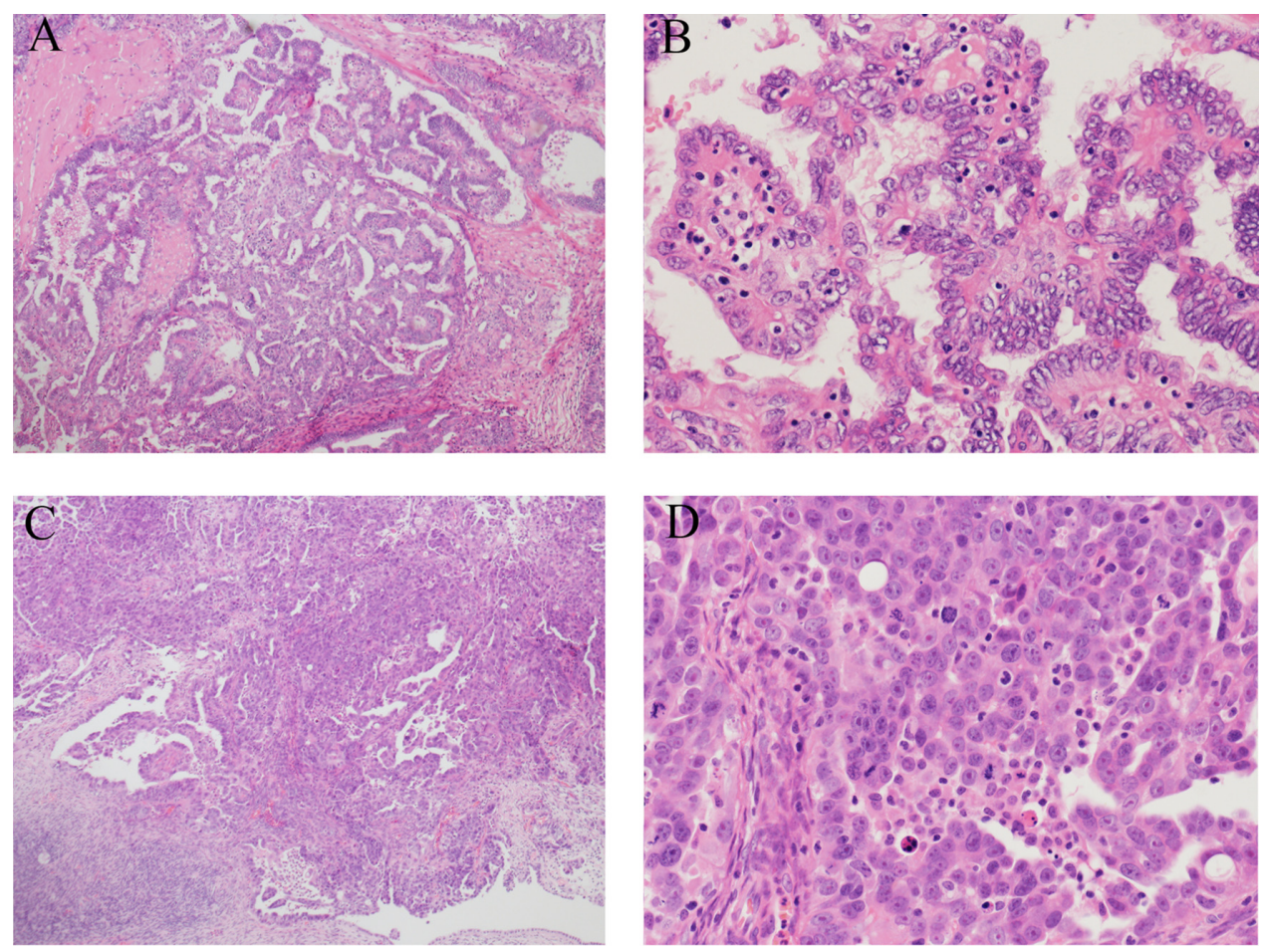

Figure I Histopathology of ovarian low-grade and high-grade serous carcinoma according to the MDACC two-tier grading system.

Notes: Low-grade serous carcinoma: uniform nuclei and infrequent mitotic figures, with low nuclear atypia of well-differentiated tumors. (A) 40x; (B) 200×. High-grade serous cancer: nuclear pleomorphism and frequent mitotic figures. Nuclear atypia is characteristic of high-grade tumors. (C) $40 \times$; (D) $200 \times$.

Abbreviation: MDACC, MD Anderson Cancer Center.

(FIGO) stage, the peritoneal cytology and the presence of residual disease after primary surgery in the two groups. The FIGO stage categories were defined as early (stages I to II) or advanced (stages III to IV).

Between-group statistical analyses of the clinicopathological variables were performed using chi-squared tests. The Kaplan-Meier method was used to calculate the distribution of patient survival. The Cox proportional hazards model was used for multivariate analyses of the prognostic value of the clinical variables. Throughout the analyses, the level of 5\% was used to denote significance. SPSS 20.0 (IBM Corporation, Armonk, NY, USA) was used for all analyses.

\section{Results \\ Patients}

Between January 2007 and December 2010, 381 patients with primary SAC were enrolled in our study. The reviewers showed high consistency in their evaluations using the 2-tier system, with full agreement regarding the serous tumors of 352 (92.4\%) patients. Finally, 35 patients were confirmed as having LGSC, and 346 were confirmed as having HGSC. Thus, LGSC comprised $9.2 \%$ of the SAC in our study.

The median age of the cohort was 54 years (range, 21-86 years), 221 (58.0\%) of whom had reached postmenopausal status. The patients with LGSC were 10 years younger than those with HGSC (46 versus [vs] 56 years, $P=0.046$ ). Of the patients with LGSC, $77 \%$ underwent optimal cytoreductive surgery (residual disease $<1 \mathrm{~cm}$ defines optimal cytoreduction); this rate was similar among those with $\operatorname{HGSC}(70 \%, P=0.735)$. Table 1 shows the baseline characteristics of these patients based on their histological subtypes.

\section{Survival analysis}

After a median follow-up time of 36 (11-82) months, 313 women $(82.2 \%)$ were considered to have disease progression. The Kaplan-Meier PFS curves for those with LGSC and those with HGSC are shown in Figure 2. For patients with LGSC, 68.6\% $(\mathrm{n}=24)$ experienced a recurrence, whereas $83.5 \%$ with HGSC ( $\mathrm{n}=289)$ had a recurrence; this difference was significant $(P=0.028)$. The median PFS and OS for patients with LGSC were 35.0 months (95\% confidence interval [CI] 30.1-39.9) and 54.0 months (95\% CI 47.2-60.8), respectively, which were longer than the survival time for those with HGSC (PFS: 16.0 months, 95\% CI 15.7-16.4; $P=0.003$; OS: 35.0 months, $95 \%$ CI 32.2-37.8; $P=0.024)$. A multivariate analysis was performed for the whole cohort of patients, and the result showed that, in 
Table I Baseline characteristics of patients with ovarian lowgrade and high-grade serous carcinoma

\begin{tabular}{|c|c|c|c|c|}
\hline Characteristics & $\begin{array}{l}\text { LGSC group } \\
(\mathrm{n}=35) \\
\text { Patients, } \\
\mathrm{n}(\%)\end{array}$ & $\begin{array}{l}\text { HGSC group } \\
(n=346) \\
\text { Patients, } \\
n(\%)\end{array}$ & $\chi^{2}$ & $P$-value \\
\hline $\begin{array}{l}\text { Median age } \\
\text { (range, years) }\end{array}$ & 46 & 56 & & \\
\hline$<50 y$ & $21(60 \%)$ & II 5 (33\%) & 9.918 & $0.002^{*}$ \\
\hline$\geq 50 y$ & 14 (40\%) & $231(67 \%)$ & & \\
\hline \multicolumn{5}{|l|}{ ECOG score } \\
\hline 0 & $30(86 \%)$ & 197 (57\%) & 10.930 & $0.00 I^{*}$ \\
\hline$\geq 1$ & $5(14 \%)$ & 149 (43\%) & & \\
\hline \multicolumn{5}{|l|}{ FIGO stage } \\
\hline 1 & $4(11 \%)$ & $21(6 \%)$ & 0.166 & 0.684 \\
\hline II & $4(11 \%)$ & $69(20 \%)$ & & \\
\hline III & 27 (77\%) & $214(62 \%)$ & & \\
\hline IV & 0 & $42(12 \%)$ & & \\
\hline \multicolumn{5}{|l|}{ Tumor size } \\
\hline$<10 \mathrm{~cm}$ & $20(57 \%)$ & $232(67 \%)$ & 1.394 & 0.238 \\
\hline$\geq 10 \mathrm{~cm}$ & $15(43 \%)$ & 114 (33\%) & & \\
\hline \multicolumn{5}{|c|}{ Peritoneal cytology } \\
\hline Negative & $26(74 \%)$ & $162(47 \%)$ & 9.592 & $0.002^{*}$ \\
\hline Positive & $9(26 \%)$ & $184(53 \%)$ & & \\
\hline \multicolumn{5}{|l|}{ Residual disease } \\
\hline$\leq \mathrm{Icm}$ & 27 (77\%) & $243(70 \%)$ & 0.735 & 0.391 \\
\hline$>\mathrm{Icm}$ & $8(23 \%)$ & $103(30 \%)$ & & \\
\hline
\end{tabular}

Note: *Difference reached statistical significance.

Abbreviations: LGSC, low-grade serous carcinoma; HGSC, high-grade serous carcinoma; ECOG, Eastern Cooperative Oncology Group; FIGO, International Federation of Gynecology and Obstetrics.

addition to FIGO stage and residual disease, tumor grade remained an important prognostic factor for PFS but not OS (PFS: 16 vs 27 months (HGSC vs LGSC), hazard ratio [HR] 2.52, $P=0.022$; OS: 44 vs 56 months (HGSC vs LGSC), HR 1.56, $P=0.056$ ) (Table 2). However, the OS between these two grades pointed to a significant difference.

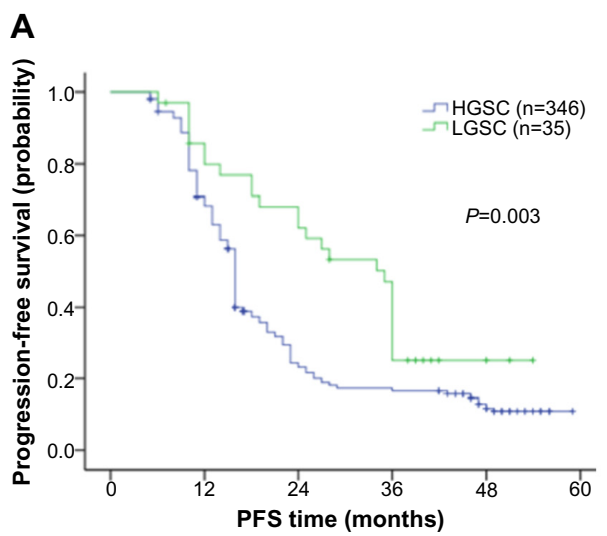

When stratified by the existence of residual disease, patients with LGSC who underwent cytoreductive surgery without a macroscopic residual $(>1 \mathrm{~cm})$ showed a significantly improved median PFS (36.0 months, 95\% CI 34.8-37.2) compared with that of patients with HGSC who received optimal cytoreductive surgery (16.0 months, $95 \%$ CI 15.1-16.9; $P=0.017$; Figure 3A). Conversely, patients with LGSC and those with HGSC who were left with a macroscopic residue $(>1 \mathrm{~cm})$ experienced a similarly worse median PFS (LGSC: 10.0 months, 95\% CI 6.6-13.4; HGSC: 13.0 months, 95\% CI 11.9-14.1; $P=0.871$; Figure $3 \mathrm{~B})$. In the OS analysis, although the difference was not significant, the patients with LGSC who underwent optimal cytoreductive surgery appeared to be associated with better OS compared with that for patients with HGSC (LGSC: 56.0 months, 95\% CI 52.3-59.7; HGSC: 44.0 months, 95\% CI 39.2-48.8; $P=0.068$; Figure 3C). No OS difference between LGSC and HGSC was observed among patients with residual disease greater than $1.0 \mathrm{~cm}$ (LGSC: 30.0 months, $95 \%$ CI $17.5-42.5$; HGSC: 28.0 months, $95 \%$ CI 25.4-30.6; $P=0.500$; Figure 3D).

Then, we performed a univariate analysis to explore the prognostic factors for PFS and OS for the LGSC and HGSC subgroups. In these two groups, FIGO stage and residual disease were significant prognostic factors for PFS and OS, and peritoneal cytology was a significant prognostic factor for PFS. Moreover, tumor size was an influential factor for the HGSC group (Table 3). After multivariate analysis of these factors was performed, however, only the FIGO stage and residual disease were significant prognostic factors (Table 4). When early stage (I-II) patients were used as the reference, the adjusted HR for disease progression and death among patients with advanced stage (III-IV)

\section{B}

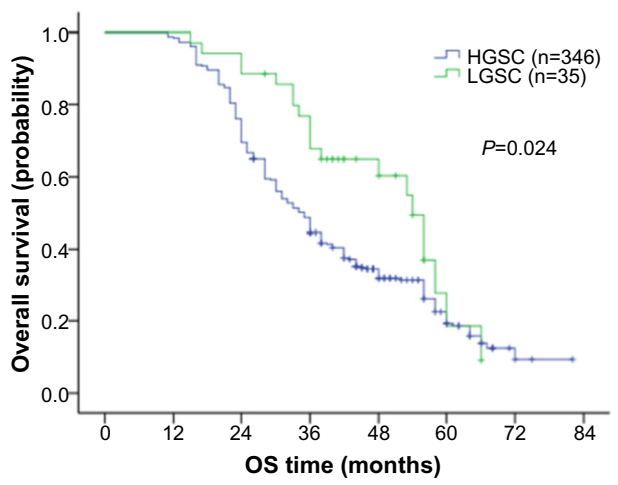

Figure 2 Comparison of progression-free and overall survival in patients with ovarian low-grade and high-grade serous carcinoma.

Notes: Progression-free curves stratified by tumor grade in the whole cohort (A); overall survival curves stratified by tumor grade in the whole cohort (B).

Abbreviations: LGSC, low-grade serous carcinoma; HGSC, high-grade serous carcinoma; PFS, progression-free survival; OS, overall survival. 
A

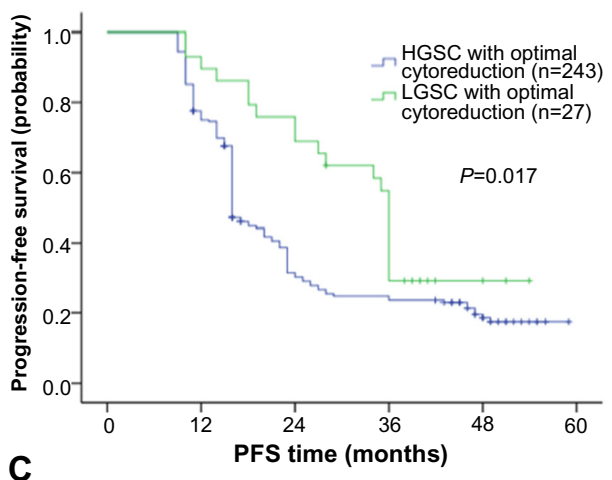

C

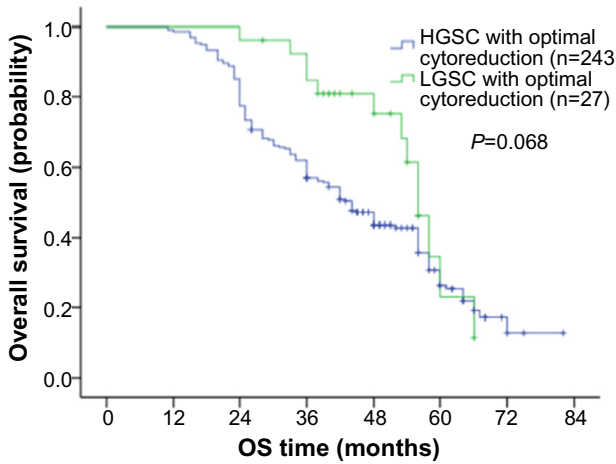

B
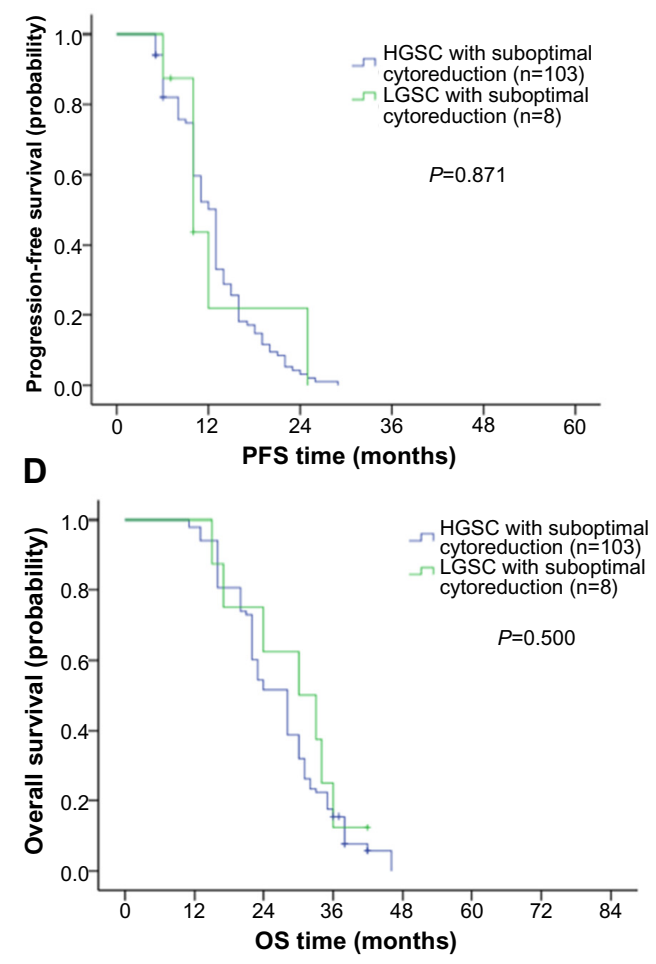

Figure 3 Comparison of progression-free and overall survival in patients stratified by tumor grade and residual disease.

Notes: Progression-free curves in patients with optimal cytoreductive surgery (A), and in patients with suboptimal cytoreductive surgery (B); overall survival curves in patients with optimal cytoreductive surgery $(\mathbf{C})$, and in patients with suboptimal cytoreductive surgery (D).

Abbreviations: LGSC, low-grade serous carcinoma; HGSC, high-grade serous carcinoma; PFS, progression-free survival; OS, overall survival.

Table 2 Multivariate regression analysis of progression-free survival and overall survival among the whole cohort $(\mathrm{n}=38 \mathrm{I})$

\begin{tabular}{|c|c|c|c|c|c|c|c|}
\hline Prognostic factors & Patients, $n$ & $\begin{array}{l}\text { Median PFS, } \\
\text { months }\end{array}$ & $\begin{array}{l}\text { PFS } \\
\text { HR }(95 \% \mathrm{Cl})\end{array}$ & $P$-value & $\begin{array}{l}\text { Median OS, } \\
\text { months }\end{array}$ & $\begin{array}{l}\text { OS } \\
\text { HR }(95 \% \mathrm{Cl})\end{array}$ & $P$-value \\
\hline \multicolumn{8}{|l|}{ Age } \\
\hline$<50 y$ & 136 & 22 & I.7I $(0.60-4.21)$ & 0.273 & 40 & $1.42(0.85-2.98)$ & 0.165 \\
\hline$\geq 50 y$ & 245 & 14 & & & 33 & & \\
\hline \multicolumn{8}{|l|}{ ECOG score } \\
\hline 0 & 227 & 18 & $1.61(0.72-2.33)$ & 0.403 & 42 & $1.26(0.78-2.54)$ & 0.225 \\
\hline$\geq 1$ & 154 & 15 & & & 36 & & \\
\hline \multicolumn{8}{|l|}{ FIGO stage } \\
\hline I-II & 98 & 24 & $3.48(1.79-5.65)$ & $<0.00 I^{*}$ & 44 & $2.53(1.48-4.76)$ & $0.043^{*}$ \\
\hline III-IV & 283 & 10 & & & 32 & & \\
\hline \multicolumn{8}{|l|}{ Grade } \\
\hline Low & 35 & 35 & $2.52(1.26-4.73)$ & $0.022^{*}$ & 54 & $1.56(0.97-2.36)$ & 0.056 \\
\hline High & 346 & 16 & & & 35 & & \\
\hline \multicolumn{8}{|l|}{ Tumor size } \\
\hline$<10 \mathrm{~cm}$ & 252 & 16 & $0.78(0.35-\mid .4 I)$ & 0.127 & 33 & $0.65(0.44-1.50)$ & 0.516 \\
\hline$\geq 10 \mathrm{~cm}$ & 129 & 18 & & & 36 & & \\
\hline \multicolumn{8}{|l|}{ Peritoneal cytology } \\
\hline Negative & 188 & 20 & $1.60(0.84-2.58)$ & 0.326 & 38 & $1.14(0.48-2.01)$ & 0.854 \\
\hline Positive & 193 & 12 & & & 32 & & \\
\hline \multicolumn{8}{|l|}{ Residual disease } \\
\hline$\leq \mathrm{Icm}$ & 270 & 17 & $3.46(1.68-5.29)$ & $<0.001 *$ & 48 & $7.04(2.55-12.43)$ & $<0.00 I^{*}$ \\
\hline$>\mathrm{Icm}$ & 111 & 13 & & & 28 & & \\
\hline
\end{tabular}

Note: *Difference reached statistical significance.

Abbreviations: LGSC, low-grade serous carcinoma; HGSC, high-grade serous carcinoma; PFS, progression-free survival; OS, overall survival; ECOG, Eastern Cooperative Oncology Group; FIGO, International Federation of Gynecology and Obstetrics; HR, hazard ratio; Cl, confidence interval. 
Table 3 Univariate regression analysis of progression-free survival and overall survival among patients with low-grade and high-grade serous carcinoma

\begin{tabular}{|c|c|c|c|c|c|c|c|c|}
\hline \multirow[t]{2}{*}{ Prognostic factors } & \multicolumn{4}{|l|}{ LGSC } & \multicolumn{4}{|l|}{ HGSC } \\
\hline & $\begin{array}{l}\text { Median PFS, } \\
\text { months }\end{array}$ & $P$-value & $\begin{array}{l}\text { Median OS, } \\
\text { months }\end{array}$ & $P$-value & $\begin{array}{l}\text { Median PFS, } \\
\text { months }\end{array}$ & $P$-value & $\begin{array}{l}\text { Median OS, } \\
\text { months }\end{array}$ & $P$-value \\
\hline \multicolumn{9}{|l|}{ Age } \\
\hline$<50 y$ & 38 & 0.676 & 62 & 0.083 & 20 & 0.365 & 40 & 0.130 \\
\hline$\geq 50 y$ & 34 & & 48 & & 14 & & 33 & \\
\hline \multicolumn{9}{|l|}{ ECOG score } \\
\hline 0 & 38 & $0.58 \mathrm{I}$ & 58 & 0.415 & 20 & $0.38 \mathrm{I}$ & 42 & 0.098 \\
\hline$\geq 1$ & 33 & & 48 & & 16 & & 33 & \\
\hline \multicolumn{9}{|l|}{ FIGO stage } \\
\hline I-II & 42 & $<0.00 I^{*}$ & 61 & $0.032^{*}$ & 22 & $<0.00 I^{*}$ & 44 & $<0.00 I^{*}$ \\
\hline III-IV & II & & 44 & & 10 & & 30 & \\
\hline \multicolumn{9}{|l|}{ Tumor size } \\
\hline$<10 \mathrm{~cm}$ & 37 & 0.513 & 60 & 0.683 & 12 & $0.043^{*}$ & 32 & 0.360 \\
\hline$\geq 10 \mathrm{~cm}$ & 32 & & 52 & & 18 & & 36 & \\
\hline \multicolumn{9}{|l|}{ Peritoneal cytology } \\
\hline Negative & 40 & $<0.00 I^{*}$ & 58 & $0.27 I$ & 20 & $0.047^{*}$ & 36 & 0.574 \\
\hline Positive & 12 & & 46 & & 14 & & 32 & \\
\hline \multicolumn{9}{|l|}{ Residual disease } \\
\hline$\leq \mathrm{I} \mathrm{cm}$ & 36 & $<0.001 *$ & 56 & $<0.001 *$ & 16 & $0.003^{*}$ & 44 & $<0.001 *$ \\
\hline$>1 \mathrm{~cm}$ & 10 & & 30 & & 13 & & 28 & \\
\hline
\end{tabular}

Note: *Difference reached statistical significance.

Abbreviations: LGSC, low-grade serous carcinoma; HGSC, high-grade serous carcinoma; PFS, progression-free survival; OS, overall survival; ECOG, Eastern Cooperative Oncology Group; FIGO, International Federation of Gynecology and Obstetrics.

diseases was 2.78 and 3.68 , respectively, for the LGSC group (PFS: 95\% CI 1.64-5.08, $P=0.016$; OS: 95\% CI $1.89-5.47, P=0.006)$. When patients without macroscopic residual disease were used as the reference, the adjusted HR for disease progression and death in those with more than $1 \mathrm{~cm}$ of residual disease was 3.89 and 3.23 , respectively, for the LGSC group (PFS: 95\% CI 1.75-4.76; $P=0.003$; OS: $95 \%$ CI $1.63-5.54, P<0.001)$. Positive ascites was also a risk factor for a shorter PFS for the patients with LGSC (HR 2.66, 95\% CI 1.80-4.62; $P=0.012$ ).

\section{Discussion}

Ovarian LGSC is a rarer disease than high-grade carcinoma, but its biological behavior has not been clarified yet. In this study, we used the MDACC two-tier system to retrospectively analyze a large ovarian cancer population to investigate the prognostic and clinical implication of LGSC. Our study demonstrates that patients with lowgrade serous ovarian cancer had a significantly younger age at diagnosis ( 46 vs 56 years, $P=0.046$ ) and that the histological grade was a significant prognostic factor for PFS but not for OS ( $P=0.021$ and $P=0.0566$, respectively). Patients with low-grade and high-grade carcinoma who were left with macroscopic residual $(>1 \mathrm{~cm})$ experienced a similar median PFS (10.0 and 13.0 months, respectively,
$P=0.871)$. This result indicates the existence of chemoresistance with LGSC. Additionally, FIGO stage and residual disease were significant prognostic factors for PFS and OS in low-grade carcinoma, while positive ascites was associated with worse PFS.

The incidence of high-grade disease was greater than that of low-grade disease among patients of all ages. ${ }^{13}$ LGSC is relatively rare, accounts for only $2 \%-10 \%$ of all serous ovarian carcinomas, and is observed less frequently in Asia. ${ }^{14-16}$ The LGSC prevalence was $9.2 \%$ in our study. LGSC usually occurs among younger women, which suggests that different clinical courses should be taken for patients with low-grade vs those with high-grade disease. ${ }^{13}$ Data from the Gynecologic Oncology Group (GOG) 182 demonstrated that the mean age of patients with LGSC was 56.6 years, whereas those with HGSC was 59.3 years old. ${ }^{17}$ The median ages of the LGSC and HGSC groups in our cohort were 46 and 56 years old, respectively, which is younger than the GOG 182 ages but similar to those measured by Schlumbrecht et al (44.9 years old) and Gershenson et al (43 years old). ${ }^{18,19}$ Although LGSC likely originates from precursor lesions in a slow stepwise fashion, ${ }^{20}$ the early-stage cases of LGSC were no more common than those of HGSC in our study ( $22 \%$ vs $26 \%$ ), which is consistent with previous studies reporting percentages of LGSC 
Table 4 Multivariate regression analysis of progression-free survival and overall survival among patients with low-grade and high-grade serous carcinoma

\begin{tabular}{|c|c|c|c|c|c|c|c|c|}
\hline \multirow[t]{3}{*}{ Prognostic factors } & \multicolumn{4}{|l|}{ LGSC } & \multicolumn{4}{|l|}{ HGSC } \\
\hline & PFS & $P$-value & OS & $P$-value & PFS & $P$-value & OS & $P$-value \\
\hline & HR (95\% Cl) & & HR (95\% Cl) & & HR (95\% Cl) & & HR (95\% Cl) & \\
\hline \multicolumn{9}{|l|}{ Age } \\
\hline$<50 y$ & $1.05(0.59-2.1)$ & 0.874 & $1.33(0.57-2.10)$ & 0.463 & $1.79(0.63-4.67)$ & 0.213 & $1.46(0.83-3.12)$ & 0.183 \\
\hline \multicolumn{9}{|l|}{$\geq 50 y$} \\
\hline \multicolumn{9}{|l|}{ ECOG score } \\
\hline 0 & $1.24(0.76-2.68)$ & 0.745 & I.I2(0.64-I.84) & 0.682 & $1.52(0.5 \mathrm{I}-1.98)$ & 0.465 & $1.56(0.82-2.76)$ & 0.160 \\
\hline \multicolumn{9}{|l|}{$\geq 1$} \\
\hline \multicolumn{9}{|l|}{ FIGO stage } \\
\hline I-II & $2.78(1.64-5.08)$ & $0.016 *$ & $2.42(1.24-4.08)$ & $0.026 *$ & $3.68(1.89-5.47)$ & $0.006 *$ & $2.89(1.54-4.68)$ & $0.01 I^{*}$ \\
\hline \multicolumn{9}{|l|}{ III-IV } \\
\hline \multicolumn{9}{|l|}{ Tumor size } \\
\hline$<10 \mathrm{~cm}$ & I.II (0.78-I.53) & 0.736 & $1.20(0.72-1.56)$ & 0.774 & $0.67(0.27-1.32)$ & 0.204 & $0.74(0.35-1.48)$ & 0.451 \\
\hline \multicolumn{9}{|l|}{$\geq 10 \mathrm{~cm}$} \\
\hline \multicolumn{9}{|l|}{ Peritoneal cytology } \\
\hline Negative & $2.66(1.80-4.62)$ & $0.012^{*}$ & $1.74(0.82-2.54)$ & 0.257 & $1.43(0.82-2.35)$ & 0.564 & $1.24(0.43-1.90)$ & 0.712 \\
\hline \multicolumn{9}{|l|}{ Positive } \\
\hline \multicolumn{9}{|l|}{ Residual disease } \\
\hline$\leq \mathrm{lcm}$ & $3.89(1.75-4.76)$ & $0.003 *$ & $3.70(1.56-5.76)$ & $<0.00 I^{*}$ & $3.23(1.63-5.54)$ & $<0.001 *$ & $7.42(2.68-11.67)$ & $<0.00 I^{*}$ \\
\hline$>1 \mathrm{~cm}$ & & & & & & & & \\
\hline
\end{tabular}

Note: "Difference reached statistical significance.

Abbreviations: LGSC, low-grade serous carcinoma; HGSC, high-grade serous carcinoma; PFS, progression-free survival; OS, overall survival; ECOG, Eastern Cooperative Oncology Group; FIGO, International Federation of Gynecology and Obstetrics; HR, hazard ratio; Cl, confidence interval.

ranging from zero to $33 \% \cdot{ }^{18,21,22}$ The reason for this result might be the lack of symptoms or signs at presentation and the failure of early detection.

Recently, many publications have supported the hypothesis that LGSC is a different entity from HGSC. LGSC likely arises from the ovarian surface epithelium, whereas HGSC arises from the fallopian tube epithelium in the majority of cases, resulting in different biological behaviors and prognoses. ${ }^{23}$ LGSCs are slow-growing tumors, as evidenced by their low mitotic figure content. ${ }^{24} \mathrm{O}^{\prime}$ Neill et al found that LGSC had a significantly lower frequency of expression of oncogenes, such as MIB1, BCL2, C-KIT, and HER2/neu, than that of HGSCs, resulting in an indolent clinical course. ${ }^{25}$ For our cohort, we observed that the LGSC group had a better survival than that of the HGSC group and that the histological grade was a prognostic factor for PFS. LGSC was associated with better OS, although this difference in the multivariate analysis was not statistically significant $(P=0.056)$, most likely due to the small sample size for LGSC. The survival advantage of LGSC has been discussed in previous studies. Bodurka et al analyzed data from GOG 158 that included 21 patients with LGSC and 220 patients with HGSC. ${ }^{26}$ Women with low-grade tumors had significantly longer median PFS values than those with high-grade carcinomas (45.0 vs 19.8 months; $P<0.001$ ). Ali et al found that LGSC showed a protracted course, but its overall disease-specific survival rate was equivalent to HGSC (4.77 vs 3.94 years, $P=0.56) .{ }^{22}$ Another study conducted at MDACC enrolled 50 patients with LGSC and 50 with HGSC. The median OS for patients with LGSC and HGSC was strikingly different (OS 4.2 vs 1.7 years, $P=0.0401$ ). ${ }^{11}$ Their study group was preferentially enriched with low-grade tumors at a 50/50 grade distribution, making it easier to reach a statistically significant difference. Importantly, certain studies have argued that the better outcomes associated with LGSC are attributable to its intrinsic biology that might allow for easier removal of the tumors; ; ${ }^{27,28}$ however, the rates of optimal cytoreductive surgery between LGSC and HGSC did not differ according to our data ( $70 \%$ vs $77 \%, P=0.691)$. Thus, we believe that LGSC is associated with better survival due to its less pernicious growth behavior.

With suboptimal cytoreduction surgery, however, a PFS or OS advantage was not observed for patients with LGSC compared with those with HGSC. In contrast, patients with LGSC who received an optimal operation had a much longer PFS (more than twice that of those with HGSC: 36 vs 16 months, $P=0.017$ ) and better OS, which indicated a significant difference (54 vs 35 months, $P=0.068$ ). This result is consistent with that of Fader, who analyzed one cohort that included 189 patients with low-grade serous cancers 
and found a PFS and OS advantage for LGSC relative to HGSC under the condition of no gross residual disease after surgery (PFS: 33.2 vs 26.8 months, OS: 96.9 vs 77.1 months, $P<0.001) .{ }^{17}$ In contrast, those with grade 1 and higher-grade serous carcinoma with measurable residual disease after surgery had a similar PFS (14.11 vs 14.39 months) and OS values (42.02 vs 37.68 months). These results suggest that optimal cytoreductive surgery has a more important effect on the survival of patients with LGSC because they receive less benefit from adjuvant chemotherapy. Many retrospective studies and laboratory data have demonstrated the chemoresistant effect of LGSC. Gershenson et al reported an overall chemotherapy response rate of $3.7 \%$ among 58 patients with recurrent low-grade serous ovarian carcinoma. ${ }^{29}$ Conversely, the response rates of taxane-platinum drug combinations among patients with HGSC ranged from $66 \%$ to $90 \% .^{30,31}$ A filtered probeset analysis demonstrated that LGSC was associated with the overexpression of KLF4 and its target CDKN1A (p21/WAF1) compared with HGSC. These proteins have both tumor suppressive and potentially oncogenic effects. ${ }^{32}$ The key role of these proteins in the inhibition of tumor proliferation might contribute to the weaker response of patients with LGSC to standard chemotherapy. ${ }^{33}$ Furthermore, a novel LGSC-associated gene, Clusterin $(C L U)$ was identified as an upregulated gene in patients with LGSC. CLU encodes for a secreted protein that was previously shown to prevent paclitaxel-induced apoptosis of ovarian cancer cell lines. Secretory $C L U$ expression was upregulated in paclitaxel-resistant ovarian cancer cells but not parental cells. Moreover, targeting CLU expression might sensitize ovarian cancer cells to paclitaxel. ${ }^{34}$

Nevertheless, peritoneal cytology was an independent prognostic factor of shorter PFS among patients with LGSC but not those with HGSC $(P=0.012$ and $P=0.564$, respectively). Our cohort merits attention. Previous studies have demonstrated that positive peritoneal washing was associated with a poorer prognosis among patients with ovarian cancers. ${ }^{35,36}$ Because positive peritoneal washing suggests the existence of intra-abdominal micro-dissemination, including chemoresistant clones, these results again imply that LGSC is relatively insensitive to conventional platinum-taxane regimens.

The weaknesses of the current study include the fact that the data were retrospectively collected, and selection bias was unavoidable. The study's strengths include the fact that our tumor specimens had undergone a pathology review using the MDACC 2-tier system and study participants received homogeneous adjuvant chemotherapies.
In conclusion, ovarian LGSC is a relatively rare disease that occurs at a younger age than the age for HGSC. The overall PFS rate of LGSC is better than that of its high-grade counterpart. However, the risk for progression among women who are left with gross residual disease after primary cytoreductive surgery is identical to that of those with HGSC. Given the chemoresistance of LGSC to conventional platinum-taxane regimens, primary cytoreductive surgery among women with LGSC is important. Future studies should explore potentially active cytotoxic effects and target drugs.

\section{Acknowledgments}

We thank the members of the Department of Pathology, Peking Union Medical College, for their help with the pathological reviews in this study.

\section{Disclosure}

The authors have no competing financial interests or conflicts of interest to disclose.

\section{References}

1. Salani R, Bristow RE. Surgical management of epithelial ovarian cancer. Clin Obstet Gynecol. 2012;55(1):75-95.

2. Kurman RJ, Shih IeM. The origin and pathogenesis of epithelial ovarian cancer: a proposed unifying theory. Am J Surg Pathol. 2010;34(3):433-443.

3. Shih IeM, Kurman RJ. Ovarian tumorigenesis: a proposed model based on morphological and molecular genetic analysis. Am J Pathol. 2004;164(5):1511-1518.

4. Seidman JD, Horkayne-Szakaly I, Cosin JA, et al. Testing of two binary grading systems for FIGO stage III serous carcinoma of the ovary and peritoneum. Gynecol Oncol. 2006;103(2):703-708.

5. Zheng JP, Robinson WR, Ehlen T, Yu MC, Dubeau L. Distinction of low grade from high grade human ovarian carcinomas on the basis of losses of heterozygosity on chromosomes 3, 6, and 11 and HER-2/neu gene amplification. Cancer Res. 1991;51(15):4045-4051.

6. Trope C, Kaern J. Adjuvant chemotherapy for early-stage ovarian cancer: review of the literature. J Clin Oncol. 2007;25(20):2909-2920.

7. Hannibal CG, Vang R, Junge J, Kjaerbye-Thygesen A, Kurman RJ, Kjaer SK. A binary histologic grading system for ovarian serous carcinoma is an independent prognostic factor: a population-based study of 4317 women diagnosed in Denmark 1978-2006. Gynecol Oncol. 2012;125(3):655-660.

8. Schmeler KM, Gershenson DM. Low-grade serous ovarian cancer: a unique disease. Curr Oncol Rep. 2008;10(6):519-523.

9. Schmeler KM, Sun CC, Bodurka DC, et al. Neoadjuvant chemotherapy for low-grade serous carcinoma of the ovary or peritoneum. Gynecol Oncol. 2008;108(3):510-514.

10. Romero I, Sun CC, Wong KK, Bast RC Jr, Gershenson DM. Low-grade serous carcinoma: new concepts and emerging therapies. Gynecol Oncol. 2013;130(3):660-666.

11. Malpica A, Deavers MT, Lu K, et al. Grading ovarian serous carcinoma using a two-tier system. Am J Surg Pathol. 2004;28(4):496-504.

12. Malpica A, Deavers MT, Tornos C, et al. Interobserver and intraobserver variability of a two-tier system for grading ovarian serous carcinoma. Am J Surg Pathol. 2007;31(8):1168-1174.

13. Plaxe SC. Epidemiology of low-grade serous ovarian cancer. $A m J$ Obstet Gynecol. 2008;198(4):459. e1-e8. 
14. Seidman JD, Horkayne-Szakaly I, Haiba M, Boice CR, Kurman RJ, Ronnett BM. The histologic type and stage distribution of ovarian carcinomas of surface epithelial origin. Int J Gynecol Pathol. 2004;23(1):41-44.

15. Diaz-Padilla I, Malpica AL, Minig L, Chiva LM, Gershenson DM, Gonzalez-Martin A. Ovarian low-grade serous carcinoma: a comprehensive update. Gynecol Oncol. 2012;126(2):279-285.

16. Carlson J, Roh MH, Chang MC, Crum CP. Recent advances in the understanding of the pathogenesis of serous carcinoma: the concept of low- and high-grade disease and the role of the fallopian tube. Diagn Histopathol (Oxf). 2008;14(8):352-365.

17. Fader AN, Java J, Ueda S, et al. Survival in women with grade 1 serous ovarian carcinoma. Obstet Gynecol. 2013;122(2 Pt 1):225-232.

18. Schlumbrecht MP, Sun CC, Wong KN, Broaddus RR, Gershenson DM, Bodurka DC. Clinicodemographic factors influencing outcomes in patients with low-grade serous ovarian carcinoma. Cancer. 2011;117(16):3741-3749.

19. Gershenson DM, Sun CC, Lu KH, et al. Clinical behavior of stage II-IV low-grade serous carcinoma of the ovary. Obstet Gynecol. 2006;108(2): 361-368.

20. May T, Shoni M, Crum CP, et al. Low-grade and high-grade serous Mullerian carcinoma: review and analysis of publicly available gene expression profiles. Gynecol Oncol. 2013;128(3):488-492.

21. Kobel M, Kalloger SE, Huntsman DG, et al. Differences in tumor type in low-stage versus high-stage ovarian carcinomas. Int J Gynecol Pathol. 2010;29(3):203-211.

22. Ali RH, Kalloger SE, Santos JL, Swenerton KD, Gilks CB. Stage II to IV low-grade serous carcinoma of the ovary is associated with a poor prognosis: a clinicopathologic study of 32 patients from a populationbased tumor registry. Int J Gynecol Pathol. 2013;32(6):529-535.

23. Crum CP, Drapkin R, Miron A, et al. The distal fallopian tube: a new model for pelvic serous carcinogenesis. Curr Opin Obstet Gynecol. 2007;19(1):3-9.

24. Lachance JA, Shutter J, Atkins KA, Stoler MH, Rice LW, Jazaeri AA. Utilization of a uniform grading system for interpreting serous ovarian cancer. Am J Obstet Gynecol. 2008;199(2):189. e1-e6.

25. O’Neill CJ, Deavers MT, Malpica A, Foster H, McCluggage WG. An immunohistochemical comparison between low-grade and highgrade ovarian serous carcinomas: significantly higher expression of p53, MIB1, BCL2, HER-2/neu, and C-KIT in high-grade neoplasms. Am J Surg Pathol. 2005;29(8):1034-1041.
26. Bodurka DC, Deavers MT, Tian C, et al. Reclassification of serous ovarian carcinoma by a 2-tier system: a Gynecologic Oncology Group Study. Cancer. 2012;118(12):3087-3094.

27. Chi DS, Schwartz PE. Cytoreduction vs neoadjuvant chemotherapy for ovarian cancer. Gynecol Oncol. 2008;111(3):391-399.

28. Borley J, Wilhelm-Benartzi C, Brown R, Ghaem-Maghami S. Does tumour biology determine surgical success in the treatment of epithelial ovarian cancer? A systematic literature review. Br J Cancer. 2012;107(7):1069-1074.

29. Gershenson DM, Sun CC, Bodurka D, et al. Recurrent low-grade serous ovarian carcinoma is relatively chemoresistant. Gynecol Oncol. 2009;114(1):48-52.

30. Rose PG, Blessing JA, Mayer AR, Homesley HD. Prolonged oral etoposide as second-line therapy for platinum-resistant and platinumsensitive ovarian carcinoma: a Gynecologic Oncology Group study. J Clin Oncol. 1998;16(2):405-410.

31. Buda A, Floriani I, Rossi R, et al. Randomised controlled trial comparing single agent paclitaxel vs epidoxorubicin plus paclitaxel in patients with advanced ovarian cancer in early progression after platinumbased chemotherapy: an Italian Collaborative Study from the Mario Negri Institute, Milan, GONO (Gruppo Oncologico Nord Ovest) group and IOR (Istituto Oncologico Romagnolo) group. Br J Cancer. 2004;90(11):2112-2117.

32. Rowland BD, Peeper DS. KLF4, p21 and context-dependent opposing forces in cancer. Nat Rev Cancer. 2006;6(1):11-23.

33. Park DC, Yeo SG, Wilson MR, et al. Clusterin interacts with Paclitaxel and confer Paclitaxel resistance in ovarian cancer. Neoplasia. 2008;10(9):964-972.

34. Hassan MK, Watari H, Han Y, et al. Clusterin is a potential molecular predictor for ovarian cancer patient's survival: targeting clusterin improves response to paclitaxel. J Exp Clin Cancer Res. 2011; 12(30):113.

35. Ahmed N, Stenvers KL. Getting to Know Ovarian Cancer Ascites: Opportunities for Targeted Therapy-Based Translational Research. Front Oncol. 2013;9(3):256.

36. Takano M, Sugiyama T, Yaegashi N, et al. The impact of complete surgical staging upon survival in early-stage ovarian clear cell carcinoma: a multi-institutional retrospective study. Int $J$ Gynecol Cancer. 2009;19(8):1353-1357.
OncoTargets and Therapy

\section{Publish your work in this journal}

OncoTargets and Therapy is an international, peer-reviewed, open access journal focusing on the pathological basis of all cancers, potential targets for therapy and treatment protocols employed to improve the management of cancer patients. The journal also focuses on the impact of management programs and new therapeutic agents and protocols on

\section{Dovepress}

patient perspectives such as quality of life, adherence and satisfaction The manuscript management system is completely online and includes a very quick and fair peer-review system, which is all easy to use. Visit http://www.dovepress.com/testimonials.php to read real quotes from published authors. 